\title{
Some New Volterra-Fredholm-Type Discrete Inequalities and Their Applications in the Theory of Difference Equations
}

\author{
Bin Zheng1 and Qinghua Feng', \\ ${ }^{1}$ School of Science, Shandong University of Technology, Shandong, Zibo 255049, China \\ 2 School of Mathematical Sciences, Qufu Normal University, Shandong, Qufu 273165, China \\ Correspondence should be addressed to Bin Zheng, zhengbin2601@126.com
}

Received 21 March 2011; Accepted 29 June 2011

Academic Editor: Martin D. Schechter

Copyright (C) 2011 B. Zheng and Q. Feng. This is an open access article distributed under the Creative Commons Attribution License, which permits unrestricted use, distribution, and reproduction in any medium, provided the original work is properly cited.

Some new Volterra-Fredholm-type discrete inequalities in two independent variables are established, which provide a handy tool in the study of qualitative and quantitative properties of solutions of certain difference equations. The established results extend some known results in the literature.

\section{Introduction}

In the research of solutions of certain differential and difference equations, if the solutions are unknown, then it is necessary to study their qualitative and quantitative properties such as boundedness, uniqueness, and continuous dependence on initial data. The GronwallBellman inequality $[1,2]$ and its various generalizations which provide explicit bounds play a fundamental role in the research of this domain. Many such generalized inequalities (e.g., see [3-30] and the references therein) have been established in the literature including the known Ou-Liang's inequality [3]. In [8], Ma generalized the discrete version of Ou-Liang's inequality in two variables to Volterra-Fredholm form for the first time, which has proved to be very useful in the study of qualitative as well as quantitative properties of solutions of certain Volterra-Fredholm-type difference equations. But since then, few results on VolterraFredholm-type discrete inequalities have been established. Recent results in this direction include the work of Ma [9] to our knowledge. We notice, in the analysis of some certain Volterra-Fredholm-type difference equations with more complicated forms, that the bounds provided by the earlier inequalities are inadequate and it is necessary to seek some new Volterra-Fredholm-type discrete inequalities in order to obtain a diversity of desired results. 
Our aim in this paper is to establish some new generalized Volterra-Fredholm-type discrete inequalities, which extend Ma's work in [9], and provide new bounds for unknown functions lying in these inequalities. We will illustrate the usefulness of the established results by applying them to study the boundedness, uniqueness, and continuous dependence on initial data of solutions of certain more complicated Volterra-Fredholm-type difference equations.

Throughout this paper, $\mathbb{R}$ denotes the set of real numbers $\mathbb{R}_{+}=[0, \infty)$, and $\mathbb{Z}$ denotes the set of integers, while $\mathbb{N}_{0}$ denotes the set of nonnegative integers. Let $\Omega:=\left(\left[m_{0}, M\right] \times\right.$ $\left.\left[n_{0}, N\right]\right) \cap \mathbb{Z}^{2}$, where $m_{0}, n_{0} \in \mathbb{Z}$ and $M, N \in \mathbb{Z} \bigcup\{\infty\}$ are two constants. $l_{1}, l_{2} \in \mathbb{Z}$ are two constants, and $K_{i}>0, i=1,2,3,4$, are all constants. If $U$ is a lattice, then we denote the set of all $\mathbb{R}$-valued functions on $U$ by $\wp(U)$ and denote the set of all $\mathbb{R}_{+}$-valued functions on $U$ by $\wp_{+}(U)$. Finally, for a function $f \in \wp_{+}(U)$, we have $\sum_{s=m_{0}}^{m_{1}} f=0$ provided $m_{0}>m_{1}$.

\section{Main Results}

Lemma 2.1 (see [15]). Assume that $a \geq 0, p \geq q \geq 0$, and $p \neq 0$ then for any $K>0$

$$
a^{q / p} \leq \frac{q}{p} K^{(q-p) / p} a+\frac{p-q}{p} K^{q / p}
$$

Lemma 2.2. Suppose that $u(m, n) \in \wp_{+}(\Omega), b(s, t, m, n) \in \wp_{+}\left(\Omega^{2}\right), \alpha \geq 0$ is a constant. If $b$ is nondecreasing in the third variable, then, for $(m, n) \in \Omega$,

$$
u(m, n) \leq \alpha+\sum_{s=m_{0}}^{m-1} \sum_{t=n_{0}}^{n-1} b(s, t, m, n) u(s, t)
$$

implies that

$$
u(m, n) \leq \alpha \exp \left\{\sum_{s=m_{0}}^{m-1} \sum_{t=n_{0}}^{n-1} b(s, t, m, n)\right\}
$$

Lemma 2.3. Suppose that $u(m, n), a(m, n), b(m, n) \in \wp_{+}(\Omega)$. If $a(m, n)$ is nondecreasing in the first variable, then, for $(m, n) \in \Omega$,

$$
u(m, n) \leq a(m, n)+\sum_{s=m_{0}}^{m-1} b(s, n) u(s, n)
$$

implies that

$$
u(m, n) \leq a(m, n) \prod_{s=m_{0}}^{m-1}[1+b(s, n)]
$$


Remark 2.4. Lemma 2.3 is a direct variation of $\left[19\right.$, Lemma $\left.2.5\left(\beta_{1}\right)\right]$, and we note $a(m, n) \geq 0$ here.

Theorem 2.5. Suppose that $u(m, n), a(m, n) \in \wp_{+}(\Omega), b_{i}(s, t, m, n), c_{i}(s, t, m, n) \in \wp_{+}\left(\Omega^{2}\right), i=$ $1,2, \ldots, l_{1}, d_{i}(s, t, m, n), e_{i}(s, t, m, n) \in \wp_{+}\left(\Omega^{2}\right), i=1,2, \ldots, l_{2}$ with $b_{i}, c_{i}, d_{i}, e_{i}$ nondecreasing in the last two variables. $p, q_{i}, r_{i}$ are nonnegative constants with $p \geq q_{i}, p \geq r_{i}, i=1,2, \ldots, l_{1}, p \neq 0$, while $h_{i}, j_{i}$ are nonnegative constants with $p \geq h_{i}, p \geq j_{i}, i=1,2, \ldots, l_{2}$. If, for $(m, n) \in \Omega, u(m, n)$ satisfies

$$
\begin{aligned}
u^{p}(m, n) \leq & a(m, n)+\sum_{i=1}^{l_{1}} \sum_{s=m_{0}}^{m-1} \sum_{t=n_{0}}^{n-1}\left[b_{i}(s, t, m, n) u^{q_{i}}(s, t)+\sum_{\xi=m_{0}}^{s} \sum_{\eta=n_{0}}^{t} c_{i}(\xi, \eta, m, n) u^{r_{i}}(\xi, \eta)\right] \\
& +\sum_{i=1}^{l_{2}} \sum_{s=m_{0}}^{M-1} \sum_{t=n_{0}}^{N-1}\left[d_{i}(s, t, m, n) u^{h_{i}}(s, t)+\sum_{\xi=m_{0}}^{s} \sum_{\eta=n_{0}}^{t} e_{i}(\xi, \eta, m, n) u^{j_{i}}(\xi, \eta)\right],
\end{aligned}
$$

then

$$
u(m, n) \leq\left\{a(m, n)+\frac{J(M, N)}{1-\mu(M, N)} C(m, n)\right\}^{1 / p}
$$

provided that $\mu(M, N)<1$, where

$$
\begin{aligned}
& J(m, n)=\sum_{i=1}^{l_{1}} \sum_{s=m_{0}}^{m-1} \sum_{t=n_{0}}^{n-1}\left\{b_{i}(s, t, m, n)\left[\frac{q_{i}}{p} K_{1}^{\left(q_{i}-p\right) / p} a(s, t)+\frac{p-q_{i}}{p} K_{1}^{q_{i} / p}\right]\right. \\
& \left.+\sum_{\xi=m_{0}}^{s} \sum_{\eta=n_{0}}^{t} c_{i}(\xi, \eta, m, n)\left[\frac{r_{i}}{p} K_{2}^{\left(r_{i}-p\right) / p} a(\xi, \eta)+\frac{p-r_{i}}{p} K_{2}^{r_{i} / p}\right]\right\} \\
& +\sum_{i=1}^{l_{2}} \sum_{s=m_{0}}^{M-1} \sum_{t=n_{0}}^{N-1}\left\{d_{i}(s, t, m, n)\left[\frac{h_{i}}{p} K_{3}^{\left(h_{i}-p\right) / p} a(s, t)+\frac{p-h_{i}}{p} K_{3}^{h_{i} / p}\right]\right. \\
& \left.+\sum_{\xi=m_{0}}^{s} \sum_{\eta=n_{0}}^{t} e_{i}(\xi, \eta, m, n)\left[\frac{j_{i}}{p} K_{4}^{\left(j_{i}-p\right) / p} a(\xi, \eta)+\frac{p-j_{i}}{p} K_{4}^{j_{i} / p}\right]\right\} \\
& \mu(m, n)=\sum_{i=1}^{l_{2}} \sum_{s=m_{0}}^{M-1} \sum_{t=n_{0}}^{N-1}\left\{d_{i}(s, t, m, n) \frac{h_{i}}{p} K_{3}^{\left(h_{i}-p\right) / p} C(s, t)+\sum_{\xi=m_{0}}^{s} \sum_{\eta=n_{0}}^{t} e_{i}(\xi, \eta, m, n) \frac{j_{i}}{p} K_{4}^{\left(j_{i}-p\right) / p} C(\xi, \eta)\right\}, \\
& C(m, n)=\exp \left\{\sum_{s=m_{0}}^{m-1} \sum_{t=n_{0}}^{n-1} B(s, t, m, n)\right\} \\
& B(s, t, m, n)=\sum_{i=1}^{l_{1}}\left[b_{i}(s, t, m, n) \frac{q_{i}}{p} K_{1}^{\left(q_{i}-p\right) / p}+\sum_{\xi=m_{0}}^{s} \sum_{\eta=n_{0}}^{t} c_{i}(\xi, \eta, m, n) \frac{r_{i}}{p} K_{2}^{\left(r_{i}-p\right) / p}\right] .
\end{aligned}
$$


Proof. Denote

$$
\begin{aligned}
v(m, n)= & \sum_{i=1}^{l_{1}} \sum_{s=m_{0}}^{m-1} \sum_{t=n_{0}}^{n-1}\left[b_{i}(s, t, m, n) u^{q_{i}}(s, t)+\sum_{\xi=m_{0}}^{s} \sum_{\eta=n_{0}}^{t} c_{i}(\xi, \eta, m, n) u^{r_{i}}(\xi, \eta)\right] \\
& +\sum_{i=1}^{l_{2}} \sum_{s=m_{0}}^{M-1} \sum_{t=n_{0}}^{N-1}\left[d_{i}(s, t, m, n) u^{h_{i}}(s, t)+\sum_{\xi=m_{0}}^{s} \sum_{\eta=n_{0}}^{t} e_{i}(\xi, \eta, m, n) u^{j_{i}}(\xi, \eta)\right]
\end{aligned}
$$

Then, we have

$$
u(m, n) \leq[a(m, n)+v(m, n)]^{1 / p}
$$

and, furthermore, from Lemma 2.1 we have

$$
\begin{aligned}
& u^{q_{i}}(m, n) \leq[a(m, n)+v(m, n)]^{q_{i} / p} \leq \frac{q_{i}}{p} K_{1}^{\left(q_{i}-p\right) / p}[a(m, n)+v(m, n)] \\
& +\frac{p-q_{i}}{p} K_{1}^{q_{i} / p}, \quad i=1,2, \ldots, l_{1} \\
& u^{r_{i}}(m, n) \leq[a(m, n)+v(m, n)]^{r_{i} / p} \leq \frac{r_{i}}{p} K_{2}^{\left(r_{i}-p\right) / p}[a(m, n)+v(m, n)] \\
& +\frac{p-r_{i}}{p} K_{2}^{r_{i} / p}, \quad i=1,2, \ldots, l_{1}, \\
& u^{h_{i}}(m, n) \leq[a(m, n)+v(m, n)]^{h_{i} / p} \leq \frac{h_{i}}{p} K_{3}^{\left(h_{i}-p\right) / p}[a(m, n)+v(m, n)] \\
& +\frac{p-h_{i}}{p} K_{3}^{h_{i} / p}, \quad i=1,2, \ldots, l_{2} \\
& u^{j_{i}}(m, n) \leq[a(m, n)+v(m, n)]^{j_{i} / p} \leq \frac{j_{i}}{p} K_{4}^{\left(j_{i}-p\right) / p}[a(m, n)+v(m, n)] \\
& +\frac{p-j_{i}}{p} K_{4}^{j_{i} / p}, \quad i=1,2, \ldots, l_{2}
\end{aligned}
$$


So

$$
\begin{aligned}
& v(m, n) \leq \sum_{i=1}^{l_{1}} \sum_{s=m_{0}}^{m-1} \sum_{t=n_{0}}^{n-1}\left\{b_{i}(s, t, m, n)\left[\frac{q_{\mathrm{i}}}{p} K_{1}^{\left(q_{i}-p\right) / p}(a(s, t)+v(s, t))+\frac{p-q_{i}}{p} K_{1}^{q_{i} / p}\right]\right. \\
& \left.+\sum_{\xi=m_{0}}^{s} \sum_{\eta=n_{0}}^{t} c_{i}(\xi, \eta, m, n)\left[\frac{r_{i}}{p} K_{2}^{\left(r_{i}-p\right) / p}(a(\xi, \eta)+v(\xi, \eta))+\frac{p-r_{i}}{p} K_{2}^{r_{i} / p}\right]\right\} \\
& +\sum_{i=1}^{l_{2}} \sum_{s=m_{0}}^{M-1} \sum_{t=n_{0}}^{N-1}\left\{d_{i}(s, t, m, n)\left[\frac{h_{i}}{p} K_{3}^{\left(h_{i}-p\right) / p}(a(s, t)+v(s, t))+\frac{p-h_{i}}{p} K_{3}^{h_{i} / p}\right]\right. \\
& \left.+\sum_{\xi=m_{0}}^{s} \sum_{\eta=n_{0}}^{t} e_{i}(\xi, \eta, m, n)\left[\frac{j_{i}}{p} K_{4}^{\left(j_{i}-p\right) / p}(a(\xi, \eta)+v(\xi, \eta))+\frac{p-j_{i}}{p} K_{4}^{j_{i} / p}\right]\right\} \\
& =H(m, n)+\sum_{i=1}^{l_{1}} \sum_{s=m_{0}}^{m-1} \sum_{t=n_{0}}^{n-1}\left[b_{i}(s, t, m, n) \frac{q_{i}}{p} K_{1}^{\left(q_{i}-p\right) / p} v(s, t)\right. \\
& \left.+\sum_{\xi=m_{0}}^{s} \sum_{\eta=n_{0}}^{t} c_{i}(\xi, \eta, m, n) \frac{r_{i}}{p} K_{2}^{\left(r_{i}-p\right) / p} v(\xi, \eta)\right],
\end{aligned}
$$

where $H(m, n)=J(m, n)+\sum_{i=1}^{l_{2}} \sum_{s=m_{0}}^{M-1} \sum_{t=n_{0}}^{N-1}\left\{d_{i}(s, t, m, n)\left(h_{i} / p\right) K_{3}^{\left(h_{i}-p\right) / p} v(s, t)+\sum_{\xi=m_{0}}^{s} \sum_{\eta=n_{0}}^{t} e_{i}\right.$ $\left.(\xi, \eta, m, n)\left(j_{i} / p\right) K_{4}^{\left(j_{i}-p\right) / p} v(\xi, \eta)\right\}$ and $J(m, n)$ is defined in (2.8). Then, using that $H(m, n)$ is nondecreasing in every variable, we obtain

$$
\begin{aligned}
& v(m, n) \leq H(M, N)+\sum_{i=1}^{l_{1}} \sum_{s=m_{0}}^{m-1} \sum_{t=n_{0}}^{n-1}\left[b_{i}(s, t, m, n) \frac{q_{i}}{p} K_{1}^{\left(q_{i}-p\right) / p} v(s, t)\right. \\
&\left.+\sum_{\xi=m_{0}}^{s} \sum_{\eta=n_{0}}^{t} c_{i}(\xi, \eta, m, n) \frac{r_{i}}{p} K_{2}^{\left(r_{i}-p\right) / p} v(\xi, \eta)\right] \\
& \leq H(M, N)+\sum_{i=1}^{l_{1}} \sum_{s=m_{0}}^{m-1} \sum_{t=n_{0}}^{n-1}\left[b_{i}(s, t, m, n) \frac{q_{i}}{p} K_{1}^{\left(q_{i}-p\right) / p}\right.\left.+\sum_{\xi=m_{0}}^{s} \sum_{\eta=n_{0}}^{t} c_{i}(\xi, \eta, m, n) \frac{r_{i}}{p} K_{2}^{\left(r_{i}-p\right) / p}\right] v(s, t) \\
&=H(M, N)+\sum_{s=m_{0}}^{m-1} \sum_{t=n_{0}}^{n-1} B(s, t, m, n) v(s, t),
\end{aligned}
$$

where $B(s, t, m, n)$ is defined in (2.11). 
Since $b_{i}(s, t, m, n), c_{i}(s, t, m, n)$ are nondecreasing in the last two variables, then $B(s, t, m, n)$ is also nondecreasing in the last two variables, and by a suitable application of Lemma 2.2 we obtain

$$
v(m, n) \leq H(M, N) \exp \left\{\sum_{s=m_{0}}^{m-1} \sum_{t=n_{0}}^{n-1} B(s, t, m, n)\right\}=H(M, N) C(m, n)
$$

where $C(m, n)$ is defined in (2.10). Furthermore, considering the definition of $H(m, n)$ and (2.17) we have

$$
\begin{aligned}
& H(M, N)=J(M, N)+\sum_{i=1}^{l_{2}} \sum_{s=m_{0}}^{M-1} \sum_{t=n_{0}}^{N-1}\left\{d_{i}(s, t, M, N) \frac{h_{i}}{p} K_{3}^{\left(h_{i}-p\right) / p} v(s, t)\right. \\
& \left.+\sum_{\xi=m_{0}}^{s} \sum_{\eta=n_{0}}^{t} e_{i}(\xi, \eta, M, N) \frac{j_{i}}{p} K_{4}^{\left(j_{i}-p\right) / p} v(\xi, \eta)\right\} \\
& \leq J(M, N)+\sum_{i=1}^{l_{2}} \sum_{s=m_{0}}^{M-1} \sum_{t=n_{0}}^{N-1}\left\{d_{i}(s, t, M, N) \frac{h_{i}}{p} K_{3}^{\left(h_{i}-p\right) / p} H(M, N) C(s, t)\right. \\
& \left.+\sum_{\xi=m_{0}}^{s} \sum_{\eta=n_{0}}^{t} e_{i}\left(\xi, \eta, m_{1}, n_{1}\right) \frac{j_{i}}{p} K_{4}^{\left(j_{i}-p\right) / p} H(M, N) C(\xi, \eta)\right\} \\
& =J(M, N)+H(M, N) \sum_{i=1}^{l_{2}} \sum_{s=m_{0}}^{M-1} \sum_{t=n_{0}}^{N-1}\left\{d_{i}(s, t, M, N) \frac{h_{i}}{p} K_{3}^{\left(h_{i}-p\right) / p} C(s, t)\right. \\
& \left.+\sum_{\xi=m_{0}}^{s} \sum_{\eta=n_{0}}^{t} e_{i}(\xi, \eta, M, N) \frac{j_{i}}{p} K_{4}^{\left(j_{i}-p\right) / p} C(\xi, \eta)\right\} \\
& =J(M, N)+H(M, N) \mu(M, N) \text {, }
\end{aligned}
$$

where $\mu(m, n)$ is defined in (2.9). Then,

$$
H(M, N) \leq \frac{J(M, N)}{1-\mu(M, N)}
$$

Combining (2.17) and (2.19) we deduce

$$
v(m, n) \leq \frac{J(M, N)}{1-\mu(M, N)} C(m, n)
$$

Then, combining (2.13) and (2.20), we obtain the desired result. 
Corollary 2.6. Suppose that $g_{1 i}(m, n), g_{2 i}(m, n), b_{1 i}(m, n), c_{1 i}(m, n) \in \wp_{+}(\Omega), i=1,2, \ldots, l_{1}$ with $g_{1 i}, g_{2 i}$ nondecreasing in every variable. $d_{1 i}(m, n), e_{1 i}(m, n) \in \wp_{+}(\Omega), i=1,2, \ldots, l_{2}$. $u(m, n), a(m, n), p, q_{i}, r_{i}, h_{i}, j_{i}$ are defined as in Theorem 2.5. If, for $(m, n) \in \Omega, u(m, n)$ satisfies

$$
\begin{aligned}
u^{p}(m, n) \leq & a(m, n)+\sum_{i=1}^{l_{1}} g_{1 i}(m, n) \sum_{s=m_{0}}^{m-1} \sum_{t=n_{0}}^{n-1}\left[b_{1 i}(s, t) u^{q_{i}}(s, t)+\sum_{\xi=m_{0}}^{s} \sum_{\eta=n_{0}}^{t} c_{1 i}(\xi, \eta) u^{r_{i}}(\xi, \eta)\right] \\
& +\sum_{i=1}^{l_{2}} g_{2 i}(m, n) \sum_{s=m_{0}}^{M-1} \sum_{t=n_{0}}^{N-1}\left[d_{1 i}(s, t) u^{h_{i}}(s, t)+\sum_{\xi=m_{0}}^{s} \sum_{\eta=n_{0}}^{t} e_{1 i}(\xi, \eta) u^{j_{i}}(\xi, \eta)\right]
\end{aligned}
$$

then

$$
u(m, n) \leq\left\{a(m, n)+\frac{J(M, N)}{1-\mu(M, N)} C(M, N)\right\}^{1 / p}
$$

provided that $\mu(M, N)<1$, where

$$
\begin{aligned}
& J(m, n)=\sum_{i=1}^{l_{1}} g_{1 i}(m, n) \sum_{s=m_{0}}^{m-1} \sum_{t=n_{0}}^{n-1}\left\{b_{1 i}(s, t)\left[\frac{q_{i}}{p} K_{1}^{\left(q_{i}-p\right) / p} a(s, t)+\frac{p-q_{i}}{p} K_{1}^{q_{i} / p}\right]\right. \\
& \left.+\sum_{\xi=m_{0}}^{s} \sum_{\eta=n_{0}}^{t} c_{1 i}(\xi, \eta)\left[\frac{r_{i}}{p} K_{2}^{\left(r_{i}-p\right) / p} a(\xi, \eta)+\frac{p-r_{i}}{p} K_{2}^{r_{i} / p}\right]\right\} \\
& +\sum_{i=1}^{l_{2}} g_{2 i}(m, n) \sum_{s=m_{0}}^{M-1} \sum_{t=n_{0}}^{N-1}\left\{d_{1 i}(s, t)\left[\frac{h_{i}}{p} K_{3}^{\left(h_{i}-p\right) / p} a(s, t)+\frac{p-h_{i}}{p} K_{3}^{h_{i} / p}\right]\right. \\
& \left.+\sum_{\xi=m_{0}}^{s} \sum_{\eta=n_{0}}^{t} e_{1 i}(\xi, \eta)\left[\frac{j_{i}}{p} K_{4}^{\left(j_{i}-p\right) / p} a(\xi, \eta)+\frac{p-j_{i}}{p} K_{4}^{j_{i} / p}\right]\right\}, \\
& \mu(m, n)=\sum_{i=1}^{l_{2}}\left\{g _ { 2 i } ( m , n ) \sum _ { s = m _ { 0 } } ^ { M - 1 } \sum _ { t = n _ { 0 } } ^ { N - 1 } \left[d_{1 i}(s, t) \frac{h_{i}}{p} K_{3}^{\left(h_{i}-p\right) / p} C(s, t)\right.\right. \\
& \left.\left.+\sum_{\xi=m_{0}}^{s} \sum_{\eta=n_{0}}^{t} e_{1 i}(\xi, \eta) \frac{j_{i}}{p} K_{4}^{\left(j_{i}-p\right) / p} C(\xi, \eta)\right]\right\} \\
& C(m, n)=\exp \left\{\sum_{s=m_{0}}^{m-1} \sum_{t=n_{0}}^{n-1} B(s, t, m, n)\right\} \\
& B(s, t, m, n)=\sum_{i=1}^{l_{1}} g_{1 i}(m, n)\left[b_{1 i}(s, t) \frac{q_{i}}{p} K_{1}^{\left(q_{i}-p\right) / p}+\sum_{\xi=m_{0}}^{s} \sum_{\eta=n_{0}}^{t} c_{1 i}(\xi, \eta) \frac{r_{i}}{p} K_{2}^{\left(r_{i}-p\right) / p}\right] .
\end{aligned}
$$


The proof of Corollary 2.6 can be completed by setting $b_{i}(s, t, m, n)=g_{1 i}(m, n) b_{1 i}(s, t)$, $c_{i}(s, t, m, n)=g_{1 i}(m, n) c_{1 i}(s, t), d_{i}(s, t, m, n)=g_{2 i}(m, n) d_{1 i}(s, t), e_{i}(s, t, m, n)=g_{2 i}(m, n) e_{1 i}(s, t)$ in Theorem 2.5.

Corollary 2.7. Suppose that $u(m, n), a(m, n), b_{i}(s, t, m, n), c_{i}(s, t, m, n), d_{i}(s, t, m, n), e_{i}(s, t, m, n)$ are defined as in Theorem 2.5. If, for $(m, n) \in \Omega, u(m, n)$ satisfies

$$
\begin{aligned}
u(m, n) \leq & a(m, n)+\sum_{i=1}^{l_{1}} \sum_{s=m_{0}}^{m-1} \sum_{t=n_{0}}^{n-1}\left[b_{i}(s, t, m, n) u(s, t)+\sum_{\xi=m_{0}}^{s} \sum_{\eta=n_{0}}^{t} c_{i}(\xi, \eta, m, n) u(\xi, \eta)\right] \\
& +\sum_{i=1}^{l_{2}} \sum_{s=m_{0}}^{M-1} \sum_{t=n_{0}}^{N-1}\left[d_{i}(s, t, m, n) u(s, t)+\sum_{\xi=m_{0}}^{s} \sum_{\eta=n_{0}}^{t} e_{i}(\xi, \eta, m, n) u(\xi, \eta)\right]
\end{aligned}
$$

then

$$
u(m, n) \leq a(m, n)+\frac{J(M, N)}{1-\mu(M, N)} C(m, n),
$$

provided that $\mu(M, N)<1$, where

$$
\begin{aligned}
J(m, n)= & \sum_{i=1}^{l_{1}} \sum_{s=m_{0}}^{m-1} \sum_{t=n_{0}}^{n-1}\left\{b_{i}(s, t, m, n) a(s, t)+\sum_{\xi=m_{0}}^{s} \sum_{\eta=n_{0}}^{t} c_{i}(\xi, \eta, m, n) a(\xi, \eta)\right\} \\
& +\sum_{i=1}^{l_{2}} \sum_{s=m_{0}}^{M-1} \sum_{t=n_{0}}^{N-1}\left\{d_{i}(s, t, m, n) a(s, t)+\sum_{\xi=m_{0}}^{s} \sum_{\eta=n_{0}}^{t} e_{i}(\xi, \eta, m, n) a(\xi, \eta)\right\}, \\
\mu(m, n)= & \sum_{i=1}^{l_{2}} \sum_{s=m_{0}}^{M-1} \sum_{t=n_{0}}^{N-1}\left\{d_{i}(s, t, m, n) C(s, t)+\sum_{\xi=m_{0}}^{s} \sum_{\eta=n_{0}}^{t} e_{i}(\xi, \eta, m, n) C(\xi, \eta)\right\}, \\
C(m, n)= & \exp \left\{\sum_{s=m_{0}}^{m-1} \sum_{t=n_{0}}^{n-1} B(s, t, m, n)\right\}, \\
B(s, t, m, n)= & \sum_{i=1}^{l_{1}}\left[b_{i}(s, t, m, n)+\sum_{\xi=m_{0}}^{s} \sum_{\eta=n_{0}}^{t} c_{i}(\xi, \eta, m, n)\right]
\end{aligned}
$$

Theorem 2.8. Suppose that $w(m, n) \in \wp_{+}(\Omega), u, a, b_{i}, c_{i}, d_{i}, e_{i}, p, q_{i}, r_{i}, h_{i}, j_{i}$ are defined as in Theorem 2.5. Furthermore, assume that $a(m, n)$ is nondecreasing in the first variable. If, for $(m, n) \in$ $\Omega, u(m, n)$ satisfies 
Abstract and Applied Analysis

$$
\begin{aligned}
u^{p}(m, n) \leq & a(m, n)+\sum_{s=m_{0}}^{m-1} w(s, n) u^{p}(m, n) \\
& +\sum_{i=1}^{l_{1}} \sum_{s=m_{0}}^{m-1} \sum_{t=n_{0}}^{n-1}\left[b_{i}(s, t, m, n) u^{q_{i}}(s, t)+\sum_{\xi=m_{0}}^{s} \sum_{\eta=n_{0}}^{t} c_{i}(\xi, \eta, m, n) u^{r_{i}}(\xi, \eta)\right] \\
& +\sum_{i=1}^{l_{2}} \sum_{s=m_{0}}^{M-1} \sum_{t=n_{0}}^{N-1}\left[d_{i}(s, t, m, n) u^{h_{i}}(s, t)+\sum_{\xi=m_{0}}^{s} \sum_{\eta=n_{0}}^{t} e_{i}(\xi, \eta, m, n) u^{j_{i}}(\xi, \eta)\right],
\end{aligned}
$$

then

$$
u(m, n) \leq\left\{\left[a(m, n)+\frac{\bar{J}(M, N)}{1-\bar{\mu}(M, N)} \bar{C}(m, n)\right] \bar{w}(m, n)\right\}^{1 / p},
$$

provided that $\bar{\mu}(M, N)<1$, where

$$
\begin{aligned}
& \bar{J}(m, n)=\sum_{i=1}^{l_{1}} \sum_{s=m_{0}}^{m-1} \sum_{t=n_{0}}^{n-1}\left\{\bar{b}_{i}(s, t, m, n)\left[\frac{q_{i}}{p} K_{1}^{\left(q_{i}-p\right) / p} a(s, t)+\frac{p-q_{i}}{p} K_{1}^{q_{i} / p}\right]\right. \\
&\left.\quad+\sum_{\xi=m_{0}}^{s} \sum_{\eta=n_{0}}^{t} \bar{c}_{i}(\xi, \eta, m, n)\left[\frac{r_{i}}{p} K_{2}^{\left(r_{i}-p\right) / p} a(\xi, \eta)+\frac{p-r_{i}}{p} K_{2}^{r_{i} / p}\right]\right\} \\
&+\sum_{i=1}^{l_{2}} \sum_{s=m_{0}}^{M-1} \sum_{t=n_{0}}^{N-1}\left\{\bar{d}_{i}(s, t, m, n)\left[\frac{h_{i}}{p} K_{3}^{\left(h_{i}-p\right) / p} a(s, t)+\frac{p-h_{i}}{p} K_{3}^{h_{i} / p}\right]\right. \\
&\left.\quad+\sum_{\xi=m_{0}}^{s} \sum_{\eta=n_{0}}^{t} \bar{e}_{i}(\xi, \eta, m, n)\left[\frac{j_{i}}{p} K_{4}^{\left(j_{i}-p\right) / p} a(\xi, \eta)+\frac{p-j_{i}}{p} K_{4}^{j_{i} / p}\right]\right\} \\
& \bar{b}_{i}(s, t, m, n)=b_{i}(s, t, m, n)(\bar{w}(s, t))^{q_{i} / p}, \\
& \bar{c}_{i}(s, t, m, n)=c_{i}(s, t, m, n)(\bar{w}(s, t))^{r_{i} / p}, \quad i=1,2, \ldots, l_{1} \\
& \bar{d}_{i}(s, t, m, n)= d_{i}(s, t, m, n)(\bar{w}(s, t))^{h_{i} / p}, \\
& \bar{e}_{i}(s, t, m, n)= e_{i}(s, t, m, n)(\bar{w}(s, t))^{j_{i} / p}, \quad i=1,2, \ldots, l_{2},
\end{aligned}
$$




$$
\begin{aligned}
\bar{w}(m, n)= & \prod_{s=m_{0}}^{m-1}[1+w(s, n)] \\
\bar{\mu}(m, n)= & \sum_{i=1}^{l_{2}} \sum_{s=m_{0}}^{M-1} \sum_{t=n_{0}}^{N-1}\left\{\bar{d}_{i}(s, t, m, n) \frac{h_{i}}{p} K_{3}^{\left(h_{i}-p\right) / p} \bar{C}(s, t)\right. \\
& \left.+\sum_{\xi=m_{0}} \sum_{\eta=n_{0}}^{t} \bar{e}_{i}(\xi, \eta, m, n) \frac{j_{i}}{p} K_{4}^{\left(j_{i}-p\right) / p} \bar{C}(\xi, \eta)\right\} \\
C(m, n)= & \exp \left\{\sum_{s=m_{0}} \sum_{t=n_{0}} \bar{B}(s, t, m, n)\right\}, \\
\bar{B}(s, t, m, n)= & \sum_{i=1}^{l_{1}}\left[\bar{b}_{i}(s, t, m, n) \frac{q_{i}}{p} K_{1}^{\left(q_{i}-p\right) / p}+\sum_{\xi=m_{0}}^{s} \sum_{\eta=n_{0}}^{t} \bar{c}_{i}(\xi, \eta, m, n) \frac{r_{i}}{p} K_{2}^{\left(r_{i}-p\right) / p}\right] .
\end{aligned}
$$

Proof. Denote

$$
\begin{aligned}
z(m, n)= & a(m, n)+\sum_{i=1}^{l_{1}} \sum_{s=m_{0}}^{m-1} \sum_{t=n_{0}}^{n-1}\left[b_{i}(s, t, m, n) u^{q_{i}}(s, t)+\sum_{\xi=m_{0}}^{s} \sum_{\eta=n_{0}}^{t} c_{i}(\xi, \eta, m, n) u^{r_{i}}(\xi, \eta)\right] \\
& +\sum_{i=1}^{l_{2}} \sum_{s=m_{0}}^{M-1} \sum_{t=n_{0}}^{N-1}\left[d_{i}(s, t, m, n) u^{h_{i}}(s, t)+\sum_{\xi=m_{0}}^{s} \sum_{\eta=n_{0}}^{t} e_{i}(\xi, \eta, m, n) u^{j_{i}}(\xi, \eta)\right] .
\end{aligned}
$$

Then, we have

$$
u^{p}(m, n) \leq z(m, n)+\sum_{s=m_{0}}^{m-1} w(s, n) u^{p}(m, n) .
$$

Obviously $z(m, n)$ is nondecreasing in the first variable. So by Lemma 2.3 we obtain

$$
u^{p}(m, n) \leq z(m, n) \prod_{s=m_{0}}^{m-1}[1+w(s, n)]=z(m, n) \bar{w}(m, n)
$$

where $\bar{w}(m, n)=\prod_{s=m_{0}}^{m-1}[1+w(s, n)]$. Define

$$
\begin{aligned}
v(m, n)= & \sum_{i=1}^{l_{1}} \sum_{s=m_{0}}^{m-1} \sum_{t=n_{0}}^{n-1}\left[b_{i}(s, t, m, n) u^{q_{i}}(s, t)+\sum_{\xi=m_{0}}^{s} \sum_{\eta=n_{0}}^{t} c_{i}(\xi, \eta, m, n) u^{r_{i}}(\xi, \eta)\right] \\
& +\sum_{i=1}^{l_{2}} \sum_{s=m_{0}}^{M-1} \sum_{t=n_{0}}^{N-1}\left[d_{i}(s, t, m, n) u^{h_{i}}(s, t)+\sum_{\xi=m_{0}}^{s} \sum_{\eta=n_{0}}^{t} e_{i}(\xi, \eta, m, n) u^{j_{i}}(\xi, \eta)\right]
\end{aligned}
$$


Then,

$$
u(m, n) \leq[(a(m, n)+v(m, n)) \bar{w}(m, n)]^{1 / p},
$$

and, furthermore, by (2.39) and Lemma 2.1 we have

$$
\begin{aligned}
& v(m, n) \leq \sum_{i=1}^{l_{1}} \sum_{s=m_{0}}^{m-1} \sum_{t=n_{0}}^{n-1}\left\{b_{i}(s, t, m, n)[(a(s, t)+v(s, t)) \bar{w}(s, t)]^{q_{i} / p}\right. \\
& \left.+\sum_{\xi=m_{0}}^{s} \sum_{\eta=n_{0}}^{t} c_{i}(\xi, \eta, m, n)[(a(\xi, \eta)+v(\xi, \eta)) \bar{w}(\xi, \eta)]^{r_{i} / p}\right\} \\
& +\sum_{i=1}^{l_{2}} \sum_{s=m_{0}}^{M-1} \sum_{t=n_{0}}^{N-1}\left\{d_{i}(s, t, m, n)[(a(s, t)+v(s, t)) \bar{w}(s, t)]^{h_{i} / p}\right. \\
& \left.+\sum_{\xi=m_{0}}^{s} \sum_{\eta=n_{0}}^{t} e_{i}(\xi, \eta, m, n)[(a(\xi, \eta)+v(\xi, \eta)) \bar{w}(\xi, \eta)]^{j_{i} / p}\right\} \\
& \leq \sum_{i=1}^{l_{1}} \sum_{s=m_{0}}^{m-1} \sum_{t=n_{0}}^{n-1}\left\{b_{i}(s, t, m, n)(\bar{w}(s, t))^{q_{i} / p}\left[\frac{q_{i}}{p} K_{1}^{\left(q_{i}-p\right) / p}(a(s, t)+v(s, t))+\frac{p-q_{i}}{p} K_{1}^{q_{i} / p}\right]\right. \\
& +\sum_{\xi=m_{0}}^{s} \sum_{\eta=n_{0}}^{t} c_{i}(\xi, \eta, m, n)(\bar{w}(\xi, \eta))^{r_{i} / p} \\
& \left.\times\left[\frac{r_{i}}{p} K_{2}^{\left(r_{i}-p\right) / p}(a(\xi, \eta)+v(\xi, \eta))+\frac{p-r_{i}}{p} K_{2}^{r_{i} / p}\right]\right\} \\
& +\sum_{i=1}^{l_{2}} \sum_{s=m_{0}}^{M-1} \sum_{t=n_{0}}^{N-1}\left\{d_{i}(s, t, m, n)(\bar{w}(s, t))^{h_{i} / p}\left[\frac{h_{i}}{p} K_{3}^{\left(q_{i}-p\right) / p}(a(s, t)+v(s, t))+\frac{p-h_{i}}{p} K_{3}^{h_{i} / p}\right]\right. \\
& +\sum_{\xi=m_{0}}^{s} \sum_{\eta=n_{0}}^{t} e_{i}(\xi, \eta, m, n)(\bar{w}(\xi, \eta))^{j_{i} / p} \\
& \left.\times\left[\frac{j_{i}}{p} K_{4}^{j_{i}-p / p}(a(\xi, \eta)+v(\xi, \eta))+\frac{p-j_{i}}{p} K_{4}^{j_{i} / p}\right]\right\} \\
& =\sum_{i=1}^{l_{1}} \sum_{s=m_{0}}^{m-1} \sum_{t=n_{0}}^{n-1}\left\{\bar{b}_{i}(s, t, m, n)\left[\frac{q_{i}}{p} K_{1}^{\left(q_{i}-p\right) / p}(a(s, t)+v(s, t))+\frac{p-q_{i}}{p} K_{1}^{q_{i} / p}\right]\right. \\
& \left.+\sum_{\xi=m_{0}}^{s} \sum_{\eta=n_{0}}^{t} \bar{c}_{i}(\xi, \eta, m, n)\left[\frac{r_{i}}{p} K_{2}^{\left(r_{i}-p\right) / p}(a(\xi, \eta)+v(\xi, \eta))+\frac{p-r_{i}}{p} K_{2}^{r_{i} / p}\right]\right\}
\end{aligned}
$$




$$
\begin{gathered}
+\sum_{i=1}^{l_{2}} \sum_{s=m_{0}}^{M-1} \sum_{t=n_{0}}^{N-1}\left\{\bar{d}_{i}(s, t, m, n)\left[\frac{h_{i}}{p} K_{3}^{\left(q_{i}-p\right) / p}(a(s, t)+v(s, t))+\frac{p-h_{i}}{p} K_{3}^{h_{i} / p}\right]\right. \\
\left.+\sum_{\xi=m_{0}}^{s} \sum_{\eta=n_{0}}^{t} \bar{e}_{i}(\xi, \eta, m, n)\left[\frac{j_{i}}{p} K_{4}^{\left(j_{i}-p\right) / p}(a(\xi, \eta)+v(\xi, \eta))+\frac{p-j_{i}}{p} K_{4}^{j_{i} / p}\right]\right\} \\
=\bar{H}(m, n)+\sum_{i=1}^{l_{1}} \sum_{s=m_{0}}^{m-1} \sum_{t=n_{0}}^{n-1}\left[\begin{array}{c}
\bar{b}_{i}(s, t, m, n) \frac{q_{i}}{p} K_{1}^{\left(q_{i}-p\right) / p} v(s, t) \\
\left.+\sum_{\xi=m_{0}}^{s} \sum_{\eta=n_{0}}^{t} \bar{c}_{i}(\xi, \eta, m, n) \frac{r_{i}}{p} K_{2}^{\left(r_{i}-p\right) / p} v(\xi, \eta)\right]
\end{array}\right.
\end{gathered}
$$

where $\bar{H}(m, n)=\bar{J}(m, n)+\sum_{i=1}^{l_{2}} \sum_{s=m_{0}}^{M-1} \sum_{t=n_{0}}^{N-1}\left\{\bar{d}_{i}(s, t, m, n)\left(h_{i} / p\right) K_{3}^{\left(h_{i}-p\right) / p} v(s, t)+\sum_{\xi=m_{0}}^{s} \sum_{\eta=n_{0}}^{t} \bar{e}_{i}\right.$ $\left.(\xi, \eta, m, n) j_{i} / p K_{4}^{\left(j_{i}-p\right) / p} v(\xi, \eta)\right\}$, and $\bar{J}(m, n), \bar{b}_{i}, \bar{c}_{i}, \bar{d}_{i}, \bar{e}_{i}$ are defined in (2.29)-(2.31) respectively.

Similar to the process of (2.15)-(2.20) we deduce

$$
v(m, n) \leq \frac{\bar{J}(M, N)}{1-\bar{\mu}(M, N)} \bar{C}(m, n)
$$

where $\bar{\mu}(m, n), \bar{C}(m, n)$ are defined in (2.32) and (2.33).

Combining (2.39) and (2.41), we get the desired result.

Remark 2.9. If we set $b_{i}(s, t, m, n)=g_{1 i}(m, n) b_{1 i}(s, t), c_{i}(s, t, m, n)=g_{1 i}(m, n) c_{1 i}(s, t), d_{i}(s, t, m, n)=$ $g_{2 i}(m, n) d_{1 i}(s, t), e_{i}(s, t, m, n)=g_{2 i}(m, n) e_{1 i}(s, t)$ or set $p=q_{i}=r_{i}=h_{i}=j_{i}=1$ in Theorem 2.8, then immediately we get two corollaries which are similar to Corollaries 2.6 and 2.7, and we omit the details for them.

Theorem 2.10. Suppose that $u, a, b_{i}, c_{i}, d_{i}, e_{i}, p, q_{i}, r_{i}, h_{i}, j_{i}$ are defined as in Theorem 2.5 . $L_{i}, T_{i}: \Omega \times \mathbb{R}_{+} \rightarrow \mathbb{R}_{+}, i=1,2, \ldots, l_{2}$, satisfies $0 \leq L_{i}(m, n, u)-L_{i}(m, n, v) \leq T_{i}(m, n, v)(u-v)$ for $u \geq v \geq 0$. If, for $(m, n) \in \Omega, u(m, n)$ satisfies

$$
\begin{aligned}
u^{p}(m, n) \leq & a(m, n)+\sum_{i=1}^{l_{1}} \sum_{s=m_{0}}^{m-1} \sum_{t=n_{0}}^{n-1}\left[b_{i}(s, t, m, n) u^{q_{i}}(s, t)+\sum_{\xi=m_{0}}^{s} \sum_{\eta=n_{0}}^{t} c_{i}(\xi, \eta, m, n) u^{r_{i}}(\xi, \eta)\right] \\
& +\sum_{i=1}^{l_{2}} \sum_{s=m_{0}}^{M-1} \sum_{t=n_{0}}^{N-1}\left[d_{i}(s, t, m, n) L_{i}\left(s, t, u^{h_{i}}(s, t)\right)+\sum_{\xi=m_{0}}^{s} \sum_{\eta=n_{0}}^{t} e_{i}(\xi, \eta, m, n) u^{j_{i}}(\xi, \eta)\right],
\end{aligned}
$$


then

$$
u(m, n) \leq\left\{a(m, n)+\frac{\widehat{J}(M, N)}{1-\widehat{\mu}(M, N)} \widehat{C}(m, n)\right\}^{1 / p}
$$

provided that $\widehat{\mu}(M, N)<1$, where

$$
\begin{aligned}
& \widehat{J}(m, n)=\sum_{i=1}^{l_{1}} \sum_{s=m_{0}}^{m-1} \sum_{t=n_{0}}^{n-1}\left\{b_{i}(s, t, m, n)\left[\frac{q_{i}}{p} K_{1}^{\left(q_{i}-p\right) / p} a(s, t)+\frac{p-q_{i}}{p} K_{1}^{q_{i} / p}\right]\right. \\
& \left.+\sum_{\xi=m_{0}}^{s} \sum_{\eta=n_{0}}^{t} c_{i}(\xi, \eta, m, n)\left[\frac{r_{i}}{p} K_{2}^{\left(r_{i}-p\right) / p} a(\xi, \eta)+\frac{p-r_{i}}{p} K_{2}^{r_{i} / p}\right]\right\} \\
& +\sum_{i=1}^{l_{2}} \sum_{s=m_{0}}^{M-1} \sum_{t=n_{0}}^{N-1}\left\{d_{i}(s, t, m, n) L_{i}\left[s, t, \frac{h_{i}}{p} K_{3}^{\left(h_{i}-p\right) / p} a(s, t)+\frac{p-h_{i}}{p} K_{3}^{h_{i} / p}\right]\right. \\
& \left.+\sum_{\xi=m_{0}}^{s} \sum_{\eta=n_{0}}^{t} e_{i}(\xi, \eta, m, n)\left[\frac{j_{i}}{p} K_{4}^{\left(j_{i}-p\right) / p} a(\xi, \eta)+\frac{p-j_{i}}{p} K_{4}^{j_{i} / p}\right]\right\}, \\
& \widehat{\mu}(m, n)=\sum_{i=1}^{l_{2}} \sum_{s=m_{0}}^{M-1} \sum_{t=n_{0}}^{N-1}\left\{\widehat{d}_{i}(s, t, m, n) \frac{h_{i}}{p} K_{3}^{\left(h_{i}-p\right) / p} C(s, t)\right. \\
& \left.+\sum_{\xi=m_{0}}^{s} \sum_{\eta=n_{0}}^{t} e_{i}(\xi, \eta, m, n) \frac{j_{i}}{p} K_{4}^{\left(j_{i}-p\right) / p} C(\xi, \eta)\right\}, \\
& \widehat{d}_{i}(s, t, m, n)=d_{i}(s, t, m, n) T_{i}\left[s, t, \frac{h_{i}}{p} K_{3}^{\left(h_{i}-p\right) / p} a(s, t)+\frac{p-h_{i}}{p} K_{3}^{h_{i} / p}\right], \quad i=1,2, \ldots, l_{2}, \\
& \widehat{C}(m, n)=\exp \left\{\sum_{s=m_{0}}^{m-1} \sum_{t=n_{0}}^{n-1} \widehat{B}(s, t, m, n)\right\} \\
& \widehat{B}(s, t, m, n)=\sum_{i=1}^{l_{1}}\left[b_{i}(s, t, m, n) \frac{q_{i}}{p} K_{1}^{\left(q_{i}-p\right) / p}+\sum_{\xi=m_{0}}^{s} \sum_{\eta=n_{0}}^{t} c_{i}(\xi, \eta, m, n) \frac{r_{i}}{p} K_{2}^{\left(r_{i}-p\right) / p}\right] .
\end{aligned}
$$


Proof. Denote

$$
\begin{aligned}
v(m, n)= & \sum_{i=1}^{l_{1}} \sum_{s=m_{0}}^{m-1} \sum_{t=n_{0}}^{n-1}\left[b_{i}(s, t, m, n) u^{q_{i}}(s, t)+\sum_{\xi=m_{0}}^{s} \sum_{\eta=n_{0}}^{t} c_{i}(\xi, \eta, m, n) u^{r_{i}}(\xi, \eta)\right] \\
& +\sum_{i=1}^{l_{2}} \sum_{s=m_{0}}^{M-1} \sum_{t=n_{0}}^{N-1}\left[d_{i}(s, t, m, n) L_{i}\left(s, t, u^{h_{i}}(s, t)\right)+\sum_{\xi=m_{0}}^{s} \sum_{\eta=n_{0}}^{t} e_{i}(\xi, \eta, m, n) u^{j_{i}}(\xi, \eta)\right] .
\end{aligned}
$$

Then

$$
u(m, n) \leq[a(m, n)+v(m, n)]^{1 / p},
$$

and, furthermore, from Lemma 2.1 we have

$$
\begin{aligned}
& v(m, n) \leq \sum_{i=1}^{l_{1}} \sum_{s=m_{0}}^{m-1} \sum_{t=n_{0}}^{n-1}\left\{b_{i}(s, t, m, n)\left[\frac{q_{i}}{p} K_{1}^{\left(q_{i}-p\right) / p}(a(s, t)+v(s, t))+\frac{p-q_{i}}{p} K_{1}^{q_{i} / p}\right]\right. \\
& \left.+\sum_{\xi=m_{0}}^{s} \sum_{\eta=n_{0}}^{t} c_{i}(\xi, \eta, m, n)\left[\frac{r_{i}}{p} K_{2}^{\left(r_{i}-p\right) / p}(a(\xi, \eta)+v(\xi, \eta))+\frac{p-r_{i}}{p} K_{2}^{r_{i} / p}\right]\right\} \\
& +\sum_{i=1}^{l_{2}} \sum_{s=m_{0}}^{M-1} \sum_{t=n_{0}}^{N-1}\left\{d_{i}(s, t, m, n) L_{i}\left[s, t, \frac{h_{i}}{p} K_{3}^{\left(h_{i}-p\right) / p}(a(s, t)+v(s, t))+\frac{p-h_{i}}{p} K_{3}^{h_{i} / p}\right]\right. \\
& \left.+\sum_{\xi=m_{0}}^{s} \sum_{\eta=n_{0}}^{t} e_{i}(\xi, \eta, m, n)\left[\frac{j_{i}}{p} K_{4}^{\left(j_{i}-p\right) / p}(a(\xi, \eta)+v(\xi, \eta))+\frac{p-j_{i}}{p} K_{4}^{j_{i} / p}\right]\right\} \\
& \leq \sum_{i=1}^{l_{1}} \sum_{s=m_{0}}^{m-1} \sum_{t=n_{0}}^{n-1}\left\{b_{i}(s, t, m, n)\left[\frac{q_{i}}{p} K_{1}^{\left(q_{i}-p\right) / p}(a(s, t)+v(s, t))+\frac{p-q_{i}}{p} K_{1}^{q_{i} / p}\right]\right. \\
& \left.+\sum_{\xi=m_{0}}^{s} \sum_{\eta=n_{0}}^{t} c_{i}(\xi, \eta, m, n)\left[\frac{r_{i}}{p} K_{2}^{\left(r_{i}-p\right) / p}(a(\xi, \eta)+v(\xi, \eta))+\frac{p-r_{i}}{p} K_{2}^{r_{i} / p}\right]\right\} \\
& +\sum_{i=1}^{l_{2}} \sum_{s=m_{0}}^{M-1} \sum_{t=n_{0}}^{N-1}\left\{d _ { i } ( s , t , m , n ) \left\{L_{i}\left[s, t, \frac{h_{i}}{p} K_{3}^{\left(h_{i}-p\right) / p}(a(s, t)+v(s, t))+\frac{p-h_{i}}{p} K_{3}^{h_{i} / p}\right]\right.\right.
\end{aligned}
$$


Abstract and Applied Analysis

$$
\begin{aligned}
& -L_{i}\left[s, t, \frac{h_{i}}{p} K_{3}^{\left(h_{i}-p\right) / p} a(s, t)+\frac{p-h_{i}}{p} K_{3}^{h_{i} / p}\right] \\
& \left.+L_{i}\left[s, t, \frac{h_{i}}{p} K_{3}^{\left(h_{i}-p\right) / p} a(s, t)+\frac{p-h_{i}}{p} K_{3}^{h_{i} / p}\right]\right\} \\
& \left.+\sum_{\xi=m_{0}}^{s} \sum_{\eta=n_{0}}^{t} e_{i}(\xi, \eta, m, n)\left[\frac{j_{i}}{p} K_{4}^{\left(j_{i}-p\right) / p}(a(\xi, \eta)+v(\xi, \eta))+\frac{p-j_{i}}{p} K_{4}^{j_{i} / p}\right]\right\} \\
& \leq \sum_{i=1}^{l_{1}} \sum_{s=m_{0}}^{m-1} \sum_{t=n_{0}}^{n-1}\left\{b_{i}(s, t, m, n)\left[\frac{q_{i}}{p} K_{1}^{\left(q_{i}-p\right) / p}(a(s, t)+v(s, t))+\frac{p-q_{i}}{p} K_{1}^{q_{i} / p}\right]\right. \\
& \left.+\sum_{\xi=m_{0}}^{s} \sum_{\eta=n_{0}}^{t} c_{i}(\xi, \eta, m, n)\left[\frac{r_{i}}{p} K_{2}^{\left(r_{i}-p\right) / p}(a(\xi, \eta)+v(\xi, \eta))+\frac{p-r_{i}}{p} K_{2}^{r_{i} / p}\right]\right\} \\
& +\sum_{i=1}^{l_{2}} \sum_{s=m_{0}}^{M-1} \sum_{t=n_{0}}^{N-1}\left\{d _ { i } ( s , t , m , n ) \left\{T_{i}\left[s, t, \frac{h_{i}}{p} K_{3}^{\left(h_{i}-p\right) / p} a(s, t)+\frac{p-h_{i}}{p} K_{3}^{h_{i} / p}\right] \frac{h_{i}}{p} K_{3}^{\left(h_{i}-p\right) / p}\right.\right. \\
& \left.\times v(s, t)+L_{i}\left[s, t, \frac{h_{i}}{p} K_{3}^{\left(h_{i}-p\right) / p} a(s, t)+\frac{p-h_{i}}{p} K_{3}^{h_{i} / p}\right]\right\} \\
& \left.+\sum_{\xi=m_{0}}^{s} \sum_{\eta=n_{0}}^{t} e_{i}(\xi, \eta, m, n)\left[\frac{j_{i}}{p} K_{4}^{\left(j_{i}-p\right) / p}(a(\xi, \eta)+v(\xi, \eta))+\frac{p-j_{i}}{p} K_{4}^{j_{i} / p}\right]\right\} \\
& =\widehat{H}(m, n)+\sum_{i=1}^{l_{1}} \sum_{s=m_{0}}^{m-1} \sum_{t=n_{0}}^{n-1}\left[b_{i}(s, t, m, n) \frac{q_{i}}{p} K_{1}^{\left(q_{i}-p\right) / p} v(s, t)\right. \\
& \left.+\sum_{\xi=m_{0}}^{s} \sum_{\eta=n_{0}}^{t} c_{i}(\xi, \eta, m, n) \frac{r_{i}}{p} K_{2}^{\left(r_{i}-p\right) / p} v(\xi, \eta)\right],
\end{aligned}
$$

where $\widehat{H}(m, n)=\widehat{J}(m, n)+\sum_{i=1}^{l_{2}} \sum_{s=m_{0}}^{M-1} \sum_{t=n_{0}}^{N-1}\left\{\widehat{d}_{i}(s, t, m, n)\left(h_{i} / p\right) K_{3}^{\left(h_{i}-p\right) / p} v(s, t)+\sum_{\xi=m_{0}}^{s} \sum_{\eta=n_{0}}^{t} e_{i}\right.$ $\left.(\xi, \eta, m, n)\left(j_{i} / p\right) K_{4}^{\left(j_{i}-p\right) / p} v(\xi, \eta)\right\}$ and $\widehat{J}(m, n), \widehat{d}_{i}(s, t, m, n)$ are defined in (2.44) and (2.46) respectively.

Similar to the process of (2.15)-(2.20) we deduce

$$
v(m, n) \leq \frac{\widehat{J}(M, N)}{1-\widehat{\mu}(M, N)} \widehat{C}(M, N),
$$

where $\widehat{\mu}(m, n), \widehat{C}(m, n)$ are defined in (2.45) and (2.47) respectively.

Combining (2.50) and (2.52), we get the desired result. 
Theorem 2.11. Suppose that $w(m, n) \in \wp_{+}(\Omega), u, a, b_{i}, c_{i}, d_{i}, e_{i}, p, q_{i}, r_{i}, h_{i}, j_{i}$ are defined as in Theorem 2.5. Furthermore, assume $a(m, n)$ is nondecreasing in the first variable. $L_{i}, T_{i}, i=$ $1,2, \ldots, l_{2}$, are defined as in Theorem 2.10. If, for $(m, n) \in \Omega, u(m, n)$ satisfies

$$
\begin{aligned}
u^{p}(m, n) \leq & a(m, n)+\sum_{s=m_{0}}^{m-1} w(s, n) u^{p}(m, n) \\
& +\sum_{i=1}^{l_{1}} \sum_{s=m_{0}}^{m-1} \sum_{t=n_{0}}^{n-1}\left[b_{i}(s, t, m, n) u^{q_{i}}(s, t)+\sum_{\xi=m_{0}}^{s} \sum_{\eta=n_{0}}^{t} c_{i}(\xi, \eta, m, n) u^{r_{i}}(\xi, \eta)\right] \\
& +\sum_{i=1}^{l_{2}} \sum_{s=m_{0}}^{M-1} \sum_{t=n_{0}}^{N-1}\left[d_{i}(s, t, m, n) L_{i}\left(s, t, u^{h_{i}}(s, t)\right)+\sum_{\xi=m_{0}}^{s} \sum_{\eta=n_{0}}^{t} e_{i}(\xi, \eta, m, n) u^{j_{i}}(\xi, \eta)\right],
\end{aligned}
$$

then

$$
u(m, n) \leq\left\{\left[a(m, n)+\frac{\tilde{J}(M, N)}{1-\widetilde{\mu}(M, N)} \widetilde{C}(m, n)\right] \widetilde{w}(m, n)\right\}^{1 / p},
$$

provided that $\tilde{\mu}(M, N)<1$, where

$$
\begin{gathered}
\tilde{J}(m, n)=\sum_{i=1}^{l_{1}} \sum_{s=m_{0}}^{m-1} \sum_{t=n_{0}}^{n-1}\left\{\tilde{b}_{i}(s, t, m, n)\left[\frac{q_{i}}{p} K_{1}^{\left(q_{i}-p\right) / p} a(s, t)+\frac{p-q_{i}}{p} K_{1}^{q_{i} / p}\right]\right. \\
\left.\quad+\sum_{\xi=m_{0}}^{s} \sum_{\eta=n_{0}}^{t} \tilde{c}_{i}(\xi, \eta, m, n)\left[\frac{r_{i}}{p} K_{2}^{\left(r_{i}-p\right) / p} a(\xi, \eta)+\frac{p-r_{i}}{p} K_{2}^{r_{i} / p}\right]\right\} \\
+\sum_{i=1}^{l_{2}} \sum_{s=m_{0}}^{M-1} \sum_{t=n_{0}}^{N-1}\left\{d_{i}(s, t, m, n) L_{i}\left[s, t,(\tilde{w}(s, t))^{h_{i} / p}\left(\frac{h_{i}}{p} K_{3}^{\left(h_{i}-p\right) / p} a(s, t)+\frac{p-h_{i}}{p} K_{3}^{h_{i} / p}\right)\right]\right. \\
\left.+\sum_{\xi=m_{0}}^{s} \sum_{\eta=n_{0}}^{t} \tilde{e}_{i}(\xi, \eta, m, n)\left[\frac{j_{i}}{p} K_{4}^{\left(j_{i}-p\right) / p} a(\xi, \eta)+\frac{p-j_{i}}{p} K_{4}^{j_{i} / p}\right]\right\},
\end{gathered}
$$

$$
\begin{aligned}
& \tilde{b}_{i}(s, t, m, n)=b_{i}(s, t, m, n)(\tilde{w}(s, t))^{q_{i} / p}, \\
& \tilde{c}_{i}(s, t, m, n)=c_{i}(s, t, m, n)(\tilde{w}(s, t))^{r_{i} / p}, \quad i=1,2, \ldots, l_{1}, \\
& \tilde{d}_{i}(s, t, m, n)=d_{i}(s, t, m, n)(\tilde{w}(s, t))^{h_{i} / p} T_{i}\left[s, t,(\tilde{w}(s, t))^{h_{i} / p}\left(\frac{h_{i}}{p} K_{3}^{\left(h_{i}-p\right) / p} a(s, t)+\frac{p-h_{i}}{p} K_{3}^{h_{i} / p}\right)\right], \\
& \tilde{e}_{i}(s, t, m, n)=e_{i}(s, t, m, n)(\tilde{w}(s, t))^{j_{i} / p}, \quad i=1,2, \ldots, l_{2},
\end{aligned}
$$




$$
\begin{aligned}
\tilde{w}(m, n)= & \prod_{s=m_{0}}^{m-1}[1+w(s, n)], \\
\tilde{\mu}(m, n)= & \sum_{i=1}^{l_{2}} \sum_{s=m_{0}}^{M-1} \sum_{t=n_{0}}^{N-1}\left\{\tilde{d}_{i}(s, t, m, n) \frac{h_{i}}{p} K_{3}^{\left(h_{i}-p\right) / p} \widetilde{C}(s, t)\right. \\
& \left.+\sum_{\xi=m_{0}}^{s} \sum_{\eta=n_{0}}^{t} \tilde{e}_{i}(\xi, \eta, m, n) \frac{j_{i}}{p} K_{4}^{\left(j_{i}-p\right) / p} \widetilde{C}(\xi, \eta)\right\}, \\
\widetilde{C}(m, n)= & \exp \left\{\sum_{s=m_{0}}^{m-1} \sum_{t=n_{0}}^{n-1} \widetilde{B}(s, t, m, n)\right\}, \\
\widetilde{B}(s, t, m, n)= & \sum_{i=1}^{l_{1}}\left[\bar{b}_{i}(s, t, m, n) \frac{q_{i}}{p} K_{1}^{\left(q_{i}-p\right) / p}+\sum_{\xi=m_{0}}^{s} \sum_{\eta=n_{0}}^{t} \bar{c}_{i}(\xi, \eta, m, n) \frac{r_{i}}{p} K_{2}^{\left(r_{i}-p\right) / p}\right] .
\end{aligned}
$$

The proof for Theorem 2.11 is similar to the combination of Theorems 2.8 and 2.10, and we omit the details here.

Remark 2.12. If we take $g_{1 i}(m, n) \equiv 1, c_{1 i}(m, n) \equiv 0, i=1,2, \ldots, l_{1}$, and $g_{2 i}(m, n) \equiv$ $1, e_{1 \mathrm{i}}(m, n) \equiv 0, \quad i=1,2, \ldots, l_{2}$ in Corollary 2.6, then Corollary 2.6 reduces to [9, Theorem 2.5]. If furthermore $l_{1}=l_{2}=1$, then Corollary 2.6 reduces to [9, Theorem 2.1]. If we take $b_{i}(s, t, m, n)=b_{1 i}(s, t), c_{i}(s, t, m, n) \equiv 0, i=1,2, \ldots, l_{1}$ and $d_{i}(s, t, m, n)=d_{1 i}(s, t)$, $e_{i}(s, t, m, n) \equiv 0, h_{i}=1, i=1,2, \ldots, l_{2}$ in Theorem 2.10, then Theorem 2.10 reduces to [ 9 , Theorem 2.7]. If furthermore $l_{1}=l_{2}=1$, then Theorem 2.10 reduces to [9, Theorem 2.6].

\section{Applications}

In this section, we will present some applications for the established results above and show that they are useful in the study of boundedness, uniqueness, and continuous dependence of solutions of certain difference equations.

Example 3.1. Consider the following Volterra-Fredholm sum-difference equation:

$$
\begin{aligned}
u^{p}(m, n)= & a(m, n)+\sum_{s=m_{0}}^{m-1} \sum_{t=n_{0}}^{n-1}\left[F_{1}(s, t, m, n, u(s, t))+\sum_{\xi=m_{0}}^{s} \sum_{\eta=n_{0}}^{t} F_{2}(\xi, \eta, m, n, u(\xi, \eta))\right] \\
& +\sum_{s=m_{0}}^{M-1} \sum_{t=n_{0}}^{M-1}\left[G_{1}(s, t, m, n, u(s, t))+\sum_{\xi=m_{0}}^{s} \sum_{\eta=n_{0}}^{t} G_{2}(\xi, \eta, m, n, u(\xi, \eta))\right],
\end{aligned}
$$


where $u(m, n), a(m, n) \in \wp(\Omega), p \geq 1$ is an odd number, $F_{i}, G_{i}: \Omega^{2} \times \mathbb{R} \rightarrow \mathbb{R}, i=1,2, M, N$ are two integers defined the same as in Theorem 2.5.

Theorem 3.2. Suppose that $u(m, n)$ is a solution of (3.1), and $\left|F_{1}(s, t, m, n, u)\right| \leq f_{1}(s, t, m, n)|u|^{q}$, $\left|F_{2}(s, t, m, n, u)\right| \leq f_{2}(s, t, m, n)|u|^{r},\left|G_{1}(s, t, m, n, u)\right| \leq g_{1}(s, t, m, n)|u|^{h},\left|G_{2}(s, t, m, n, u)\right| \leq$ $g_{2}(s, t, m, n)|u|^{j}$, where $q, r, h, j$ are nonnegative constants satisfying $p \geq q, p \geq r, p \geq h, p \geq j$, $f_{i}, g_{i} \in \wp_{+}\left(\Omega^{2}\right), i=1,2$ and $f_{i}, g_{i}$ are nondecreasing in the last two variables; then one has

$$
|u(m, n)| \leq\left\{|a(m, n)|+\frac{J(M, N)}{1-\mu(M, N)} C(m, n)\right\}^{1 / p},
$$

provided that $\mu(M, N)<1$, where

$$
\begin{aligned}
J(m, n)= & \sum_{s=m_{0}}^{m-1} \sum_{t=n_{0}}^{n-1}\left\{f_{1}(s, t, m, n)\left[\frac{q}{p} K_{1}^{(q-p) / p}|a(s, t)|+\frac{p-q}{p} K_{1}^{q / p}\right]\right. \\
& \left.\quad+\sum_{\xi=m_{0}}^{s} \sum_{\eta=n_{0}}^{t} f_{2}(\xi, \eta, m, n)\left[\frac{r}{p} K_{2}^{(r-p) / p}|a(\xi, \eta)|+\frac{p-r}{p} K_{2}^{r / p}\right]\right\} \\
& +\sum_{s=m_{0}}^{M-1} \sum_{t=n_{0}}^{N-1}\left\{g_{1}(s, t, m, n)\left[\frac{h}{p} K_{3}^{(h-p) / p}|a(s, t)|+\frac{p-h}{p} K_{3}^{h / p}\right]\right. \\
& \left.+\sum_{\xi=m_{0}}^{s} \sum_{\eta=n_{0}}^{t} g_{2}(\xi, \eta, m, n)\left[\frac{j}{p} K_{4}^{(j-p) / p}|a(\xi, \eta)|+\frac{p-j}{p} K_{4}^{j / p}\right]\right\}, \\
\mu(m, n)= & \sum_{s=m_{0}}^{M-1} \sum_{t=n_{0}}^{N-1}\left\{g_{1}(s, t, m, n) \frac{h}{p} K_{3}^{(h-p) / p} C(s, t)\right. \\
& \left.+\sum_{\xi=m_{0}}^{s} \sum_{\eta=n_{0}}^{t} g_{2}(\xi, \eta, m, n) \frac{j}{p} K_{4}^{(j-p) / p} C(\xi, \eta)\right\}, \\
B(s, t, m, n)= & f_{1}(s, t, m, n) \frac{q}{p} K_{1}^{(q-p) / p}+\sum_{\xi=m_{0}}^{s} \sum_{\eta=n_{0}}^{t} f_{2}(\xi, \eta, m, n) \frac{r}{p} K_{2}^{(r-p) / p} . \\
C(m, n)= & \exp \left\{\sum_{s=m_{0}}^{m-1} \sum_{t=n_{0}}^{n-1} B(s, t, m, n)\right\},
\end{aligned}
$$


Proof. From (3.1) we have

$$
\begin{aligned}
|u(m, n)|^{p} \leq & |a(m, n)|+\sum_{s=m_{0}}^{m-1} \sum_{t=n_{0}}^{n-1}\left[\left|F_{1}(s, t, m, n, u(s, t))\right|+\sum_{\xi=m_{0}}^{s} \sum_{\eta=n_{0}}^{t}\left|F_{2}(\xi, \eta, m, n, u(\xi, \eta))\right|\right] \\
& +\sum_{s=m_{0}}^{M-1} \sum_{t=n_{0}}^{M-1}\left[\left|G_{1}(s, t, m, n, u(s, t))\right|+\sum_{\xi=m_{0}}^{s} \sum_{\eta=n_{0}}^{t}\left|G_{2}(\xi, \eta, m, n, u(\xi, \eta))\right|\right] \\
\leq & |a(m, n)|+\sum_{s=m_{0}} \sum_{t=n_{0}}^{n-1}\left[f_{1}(s, t, m, n)|u(s, t)|^{q}+\sum_{\xi=m_{0}}^{s} \sum_{\eta=n_{0}}^{t} f_{2}(\xi, \eta, m, n)|u(\xi, \eta)|^{r}\right] \\
& +\sum_{s=m_{0}}^{M-1} \sum_{t=n_{0}}^{M-1}\left[g_{1}(s, t, m, n)|u(s, t)|^{h}+\sum_{\xi=m_{0}}^{s} \sum_{\eta=n_{0}}^{t} g_{2}(\xi, \eta, m, n)|u(\xi, \eta)|^{j}\right] .
\end{aligned}
$$

Then a suitable application of Theorem 2.5 (with $l_{1}=l_{2}=1$ ) to (3.4) yields the desired result.

The following theorem deals with the uniqueness of the solutions of (3.1).

Theorem 3.3. Suppose that $\left|F_{i}(s, t, m, n, u)-F_{i}(s, t, m, n, v)\right| \leq f_{i}(s, t, m, n)\left|u^{p}-v^{p}\right|, \mid$ $G_{i}(s, t, m, n, u)-G_{i}(s, t, m, n, v)\left|\leq g_{i}(s, t, m, n)\right| u^{p}-v^{p} \mid, i=1,2$ hold for $\forall u, v \in \mathbb{R}$, where $f_{i}, g_{i} \in \xi_{+}\left(\Omega^{2}\right), i=1,2$ with $f_{i}, g_{i}$ nondecreasing in the last two variables, and $\mu(M, N)=$ $\sum_{s=m_{0}}^{M-1} \sum_{t=n_{0}}^{N-1}\left\{g_{1}(s, t, M, N) C(s, t)+\sum_{\xi=m_{0}}^{s} \sum_{\eta=n_{0}}^{t} g_{2}(\xi, \eta, M, N) C(\xi, \eta)\right\}<1$, where $C(m, n)=$ $\exp \left\{\sum_{s=m_{0}}^{m-1} \sum_{t=n_{0}}^{n-1} B(s, t, m, n)\right\}$, and $B(s, t, m, n)=f_{1}(s, t, m, n)+\sum_{\xi=m_{0}}^{s} \sum_{\eta=n_{0}}^{t} f_{2}(\xi, \eta, m, n)$, then (3.1) has at most one solution.

Proof. Suppose that $u_{1}(m, n), u_{2}(m, n)$ are two solutions of (3.1). Then,

$$
\begin{aligned}
\left|u_{1}^{p}(m, n)-u_{2}^{p}(m, n)\right| \leq \sum_{s=m_{0}} \sum_{t=n_{0}}^{m-1}[\mid & F_{1}\left(s, t, m, n, u_{1}(s, t)\right)-F_{1}\left(s, t, m, n, u_{2}(s, t)\right) \mid \\
& \left.+\sum_{\xi=m_{0}}^{s} \sum_{\eta=n_{0}}^{t}\left|F_{2}\left(\xi, \eta, m, n, u_{1}(\xi, \eta)\right)-F_{2}\left(\xi, \eta, m, n, u_{2}(\xi, \eta)\right)\right|\right] \\
+\sum_{s=m_{0}}^{M-1} \sum_{t=n_{0}}^{M-1}\left[\left|G_{1}\left(s, t, m, n, u_{1}(s, t)\right)-G_{1}\left(s, t, m, n, u_{2}(s, t)\right)\right|\right. & \\
& \left.+\sum_{\xi=m_{0}}^{s} \sum_{\eta=n_{0}}^{t}\left|G_{2}\left(\xi, \eta, m, n, u_{1}(\xi, \eta)\right)-G_{2}\left(\xi, \eta, m, n, u_{2}(\xi, \eta)\right)\right|\right]
\end{aligned}
$$




$$
\begin{aligned}
& \leq \sum_{s=m_{0}}^{m-1} \sum_{t=n_{0}}^{n-1} {\left[f_{1}(s, t, m, n)\left|u_{1}^{p}(s, t)-u_{2}^{p}(s, t)\right|\right.} \\
&\left.\quad \sum_{\xi=m_{0}}^{s} \sum_{\eta=n_{0}}^{t} f_{2}(\xi, \eta, m, n)\left|u_{1}^{p}(\xi, \eta)-u_{2}^{p}(\xi, \eta)\right|\right] \\
&+\sum_{s=m_{0}} \sum_{t=n_{0}}^{M-1}\left[g_{1}(s, t, m, n)\left|u_{1}^{p}(s, t)-u_{2}^{p}(s, t)\right|\right. \\
&\left.\quad+\sum_{\xi=m_{0}}^{s} \sum_{\eta=n_{0}}^{t} g_{2}(\xi, \eta, m, n)\left|u_{1}^{p}(\xi, \eta)-u_{2}^{p}(\xi, \eta)\right|\right] .
\end{aligned}
$$

Treat $\left|u_{1}^{p}(m, n)-u_{2}^{p}(m, n)\right|$ as one variable, and a suitable application of Corollary 2.7 yields $\left|u_{1}^{p}(m, n)-u_{2}^{p}(m, n)\right| \leq 0$, which implies that $u_{1}^{p}(m, n) \equiv u_{2}^{p}(m, n)$. Since $p$ is an odd number, then we have $u_{1}(m, n) \equiv u_{2}(m, n)$, and the proof is complete.

Finally we study the continuous dependence of the solutions of (3.1) on the functions $a, F_{1}, F_{2}, G_{1}, G_{2}$.

Theorem 3.4. Suppose that $u(m, n)$ is a solution of (3.1), $\left|F_{i}(s, t, m, n, u)-F_{i}(s, t, m, n, v)\right| \leq$ $f_{i}(s, t, m, n)\left|u^{p}-v^{p}\right|,\left|G_{i}(s, t, m, n, u)-G_{i}(s, t, m, n, v)\right| \leq g_{i}(s, t, m, n)\left|u^{p}-v^{p}\right|, i=1,2$ hold for $\forall u, v \in \mathbb{R}$, where $f_{i}, g_{i} \in \wp_{+}\left(\Omega^{2}\right), i=1,2$ with $f_{i}, g_{i}$ nondecreasing in the last two variables, and, furthermore,

$$
\begin{aligned}
|a(m, n)-\bar{a}(m, n)|+ & \sum_{s=m_{0}}^{m-1} \sum_{t=n_{0}}^{n-1}\left\{\left|F_{1}(s, t, \bar{u}(s, t))-\bar{F}_{1}(s, t, \bar{u}(s, t))\right|\right. \\
& \left.+\sum_{\xi=m_{0}}^{s} \sum_{\eta=n_{0}}^{t}\left|F_{2}(\xi, \eta, \bar{u}(\xi, \eta))-\bar{F}_{2}(\xi, \eta, \bar{u}(\xi, \eta))\right|\right\} \\
+\sum_{s=m_{0}} \sum_{t=n_{0}}^{M-1}\left\{\left|G_{1}(s, t, \bar{u}(s, t))-\bar{G}_{1}(s, t, \bar{u}(s, t))\right|\right. & \\
& \left.+\sum_{\xi=m_{0}}^{s} \sum_{\eta=n_{0}}^{t}\left|G_{2}(\xi, \eta, \bar{u}(\xi, \eta))-\bar{G}_{2}(\xi, \eta, \bar{u}(\xi, \eta))\right|\right\} \leq \varepsilon
\end{aligned}
$$

where $\varepsilon>0$ is a constant, and $\bar{u}(m, n) \in \mathfrak{Q}(\Omega)$ is the solution of the following difference equation: 
Abstract and Applied Analysis

$$
\begin{aligned}
\bar{u}^{p}(m, n)= & \bar{a}(m, n)+\sum_{s=m_{0}}^{m-1} \sum_{t=n_{0}}^{n-1}\left[\bar{F}_{1}(s, t, m, n, \bar{u}(s, t))+\sum_{\xi=m_{0}}^{s} \sum_{\eta=n_{0}}^{t} \bar{F}_{2}(\xi, \eta, m, n, \bar{u}(\xi, \eta))\right] \\
& +\sum_{s=m_{0}}^{M-1} \sum_{t=n_{0}}^{M-1}\left[\bar{G}_{1}(s, t, m, n, \bar{u}(s, t))+\sum_{\xi=m_{0}}^{s} \sum_{\eta=n_{0}}^{t} \bar{G}_{2}(\xi, \eta, m, n, \bar{u}(\xi, \eta))\right],
\end{aligned}
$$

where $\bar{F}_{i}, \bar{G}_{i}: \Omega^{2} \times \mathbb{R} \rightarrow \mathbb{R}, i=1,2$; then one has

$$
\left|u^{p}(m, n)-\bar{u}^{p}(m, n)\right| \leq \varepsilon\left[1+\frac{J(M, N)}{1-\mu(M, N)} C(m, n)\right],
$$

provided that $\mu(M, N)<1$, where

$$
\begin{aligned}
J(m, n)= & \sum_{s=m_{0}}^{m-1} \sum_{t=n_{0}}^{n-1}\left\{f_{1}(s, t, m, n)+\sum_{\xi=m_{0}}^{s} \sum_{\eta=n_{0}}^{t} f_{2}(\xi, \eta, m, n)\right\} \\
& +\sum_{s=m_{0}}^{M-1} \sum_{t=n_{0}}^{N-1}\left\{g_{1}(s, t, m, n)+\sum_{\xi=m_{0}}^{s} \sum_{\eta=n_{0}}^{t} g_{2}(\xi, \eta, m, n)\right\}, \\
\mu(m, n)= & \sum_{s=m_{0}}^{M-1} \sum_{t=n_{0}}^{N-1}\left\{g_{1}(s, t, m, n) C(s, t)+\sum_{\xi=m_{0}}^{s} \sum_{\eta=n_{0}}^{t} g_{2}(\xi, \eta, m, n) C(\xi, \eta)\right\}, \\
C(m, n)= & \exp \left\{\sum_{s=m_{0}}^{m-1} \sum_{t=n_{0}}^{n-1} B(s, t, m, n)\right\}, \\
B(s, t, m, n)= & f_{1}(s, t, m, n)+\sum_{\xi=m_{0}}^{s} \sum_{\eta=n_{0}}^{t} f_{2}(\xi, \eta, m, n) .
\end{aligned}
$$

Proof. From (3.1) and (3.7) we deduce

$$
\begin{aligned}
& \left|u^{p}(m, n)-\bar{u}^{p}(m, n)\right| \leq|a(m, n)-\bar{a}(m, n)| \\
& +\sum_{s=m_{0}} \sum_{t=n_{0}}^{m-1}\left\{\left|F_{1}(s, t, m, n, u(s, t))-\bar{F}_{1}(s, t, m, n, \bar{u}(s, t))\right|\right. \\
& \left.\quad+\sum_{\xi=m_{0}}^{s-1} \sum_{\eta=n_{0}}^{t}\left|F_{2}(\xi, \eta, m, n, u(\xi, \eta))-\bar{F}_{2}(\xi, \eta, m, n, \bar{u}(\xi, \eta))\right|\right\}
\end{aligned}
$$




$$
\begin{aligned}
& +\sum_{s=m_{0}}^{M-1} \sum_{t=n_{0}}^{N-1}\left\{\left|G_{1}(s, t, m, n, u(s, t))-\bar{G}_{1}(s, t, m, n, \bar{u}(s, t))\right|\right. \\
& \left.+\sum_{\xi=m_{0}}^{s} \sum_{\eta=n_{0}}^{t}\left|G_{2}(\xi, \eta, m, n, u(\xi, \eta))-\bar{G}_{2}(\xi, \eta, m, n, \bar{u}(\xi, \eta))\right|\right\} \\
& \leq|a(m, n)-\bar{a}(m, n)| \\
& +\sum_{s=m_{0}}^{m-1} \sum_{t=n_{0}}^{n-1}\left\{\left|F_{1}(s, t, m, n, u(s, t))-F_{1}(s, t, m, n, \bar{u}(s, t))\right|\right. \\
& +\left|F_{1}(s, t, m, n, \bar{u}(s, t))-\bar{F}_{1}(s, t, m, n, \bar{u}(s, t))\right| \\
& +\sum_{\xi=m_{0}}^{s} \sum_{\eta=n_{0}}^{t}\left|F_{2}(\xi, \eta, m, n, u(\xi, \eta))-F_{2}(\xi, \eta, m, n, \bar{u}(\xi, \eta))\right| \\
& \left.+\left|F_{2}(\xi, \eta, m, n, \bar{u}(\xi, \eta))-\bar{F}_{2}(\xi, \eta, m, n, \bar{u}(\xi, \eta))\right|\right\} \\
& +\sum_{s=m_{0}}^{M-1} \sum_{t=n_{0}}^{N-1}\left\{\left|G_{1}(s, t, m, n, u(s, t))-G_{1}(s, t, m, n, \bar{u}(s, t))\right|\right. \\
& +\left|G_{1}(s, t, m, n, \bar{u}(s, t))-\bar{G}_{1}(s, t, m, n, \bar{u}(s, t))\right| \\
& +\sum_{\xi=m_{0}}^{s} \sum_{\eta=n_{0}}^{t}\left|G_{2}(\xi, \eta, m, n, u(\xi, \eta))-G_{2}(\xi, \eta, m, n, \bar{u}(\xi, \eta))\right| \\
& \left.+\left|G_{2}(\xi, \eta, m, n, \bar{u}(\xi, \eta))-\bar{G}_{2}(\xi, \eta, m, n, \bar{u}(\xi, \eta))\right|\right\} \\
& \leq \varepsilon+\sum_{s=m_{0}}^{m-1} \sum_{t=n_{0}}^{n-1}\left\{\left|F_{1}(s, t, m, n, u(s, t))-F_{1}(s, t, m, n, \bar{u}(s, t))\right|\right. \\
& \left.+\left|F_{2}(\xi, \eta, m, n, u(\xi, \eta))-F_{2}(\xi, \eta, m, n, \bar{u}(\xi, \eta))\right|\right\} \\
& +\sum_{s=m_{0}}^{M-1} \sum_{t=n_{0}}^{N-1}\left\{\left|G_{1}(s, t, m, n, u(s, t))-G_{1}(s, t, m, n, \bar{u}(s, t))\right|\right. \\
& \left.+\sum_{\xi=m_{0}}^{s} \sum_{\eta=n_{0}}^{t}\left|G_{2}(\xi, \eta, m, n, u(\xi, \eta))-G_{2}(\xi, \eta, m, n, \bar{u}(\xi, \eta))\right|\right\} \\
& \leq \varepsilon+\sum_{s=m_{0}}^{m-1} \sum_{t=n_{0}}^{n-1}\left[f_{1}(s, t, m, n)\left|u^{p}(s, t)-\bar{u}^{p}(s, t)\right|\right.
\end{aligned}
$$




$$
\begin{array}{r}
\left.+\sum_{\xi=m_{0}}^{s} \sum_{\eta=n_{0}}^{t} f_{2}(\xi, \eta, m, n)\left|u^{p}(\xi, \eta)-\bar{u}^{p}(\xi, \eta)\right|\right] \\
+\sum_{s=m_{0}}^{M-1} \sum_{t=n_{0}}^{M-1}\left[g_{1}(s, t, m, n)\left|u^{p}(s, t)-\bar{u}^{p}(s, t)\right|\right. \\
\left.+\sum_{\xi=m_{0}}^{s} \sum_{\eta=n_{0}}^{t} g_{2}(\xi, \eta, m, n)\left|u^{p}(\xi, \eta)-\bar{u}^{p}(\xi, \eta)\right|\right] .
\end{array}
$$

Then a suitable application of Corollary 2.7 yields the desired result.

Remark 3.5. We note that the results in [1-30] are not available here to establish the analysis above.

\section{Acknowledgment}

The authors thank the referees very much for their careful comments and valuable suggestions on this paper.

\section{References}

[1] T. H. Gronwall, "Note on the derivatives with respect to a parameter of the solutions of a system of differential equations," Annals of Mathematics, vol. 20, no. 4, pp. 292-296, 1919.

[2] R. Bellman, "The stability of solutions of linear differential equations," Duke Mathematical Journal, vol. 10, pp. 643-647, 1943.

[3] L. Ou-Liang, "The boundedness of solutions of linear differential equations $y^{\prime \prime}+A(t) y^{\prime}=0$," Advances in Mathematics, vol. 3, pp. 409-415, 1957.

[4] B. G. Pachpatte, Inequalities for Differential and Integral Equations, Academic Press, New York, NY, USA, 1998.

[5] W. S. Cheung, "Some new nonlinear inequalities and applications to boundary value problems," Nonlinear Analysis, vol. 64, no. 9, pp. 2112-2128, 2006.

[6] X. Q. Zhao, Q. X. Zhao, and F. W. Meng, "On some new nonlinear discrete inequalities and their applications," Journal of Inequalities in Pure and Applied Mathematics, vol. 7, no. 2, article 52, pp. 1-9, 2006.

[7] E. H. Yang, "On some nonlinear integral and discrete inequalities related to Ou-Iang's inequality," Acta Mathematica Sinica, vol. 14, no. 3, pp. 353-360, 1998.

[8] Q. H. Ma, "Some new nonlinear Volterra-Fredholm-type discrete inequalities and their applications," Journal of Computational and Applied Mathematics, vol. 216, no. 2, pp. 451-466, 2008.

[9] Q. H. Ma, "Estimates on some power nonlinear Volterra-Fredholm type discrete inequalities and their applications," Journal of Computational and Applied Mathematics, vol. 233, no. 9, pp. 2170-2180, 2010.

[10] Q. H. Ma and J. Pečarić, "Estimates on solutions of some new nonlinear retarded Volterra-Fredholm type integral inequalities," Nonlinear Analysis: Theory, Methods \& Applications, vol. 69, no. 2, pp. 393407, 2008.

[11] B. G. Pachpatte, "Inequalities applicable in the theory of finite difference equations," Journal of Mathematical Analysis and Applications, vol. 222, no. 2, pp. 438-459, 1998.

[12] B. G. Pachpatte, "On some new inequalities related to a certain inequality arising in the theory of differential equations," Journal of Mathematical Analysis and Applications, vol. 251, no. 2, pp. 736-751, 2000. 
[13] W. S. Cheung, Q. H. Ma, and J. Pečarić, "Some discrete nonlinear inequalities and applications to difference equations," Acta Mathematica Scientia, vol. 28, no. 2, pp. 417-430, 2008.

[14] S. F. Deng, "Nonlinear discrete inequalities with two variables and their applications," Applied Mathematics and Computation, vol. 217, no. 5, pp. 2217-2225, 2010.

[15] F. C. Jiang and F. W. Meng, "Explicit bounds on some new nonlinear integral inequalities with delay," Journal of Computational and Applied Mathematics, vol. 205, no. 1, pp. 479-486, 2007.

[16] Q. H. Ma and W. S. Cheung, "Some new nonlinear difference inequalities and their applications," Journal of Computational and Applied Mathematics, vol. 202, no. 2, pp. 339-351, 2007.

[17] Q. H. Ma, "N-independent-variable discrete inequalities of Gronwall-Ou-Iang type," Annals of Differential Equations, vol. 16, pp. 813-820, 2000.

[18] P. Y. H. Pang and R. P. Agarwal, "On an integral inequality and its discrete analogue," Journal of Mathematical Analysis and Applications, vol. 194, no. 2, pp. 569-577, 1995.

[19] B. G. Pachpatte, "On some fundamental integral inequalities and their discrete analogues," Journal of Inequalities in Pure and Applied Mathematics, vol. 2, no. 2, article 15, 2001.

[20] F. W. Meng and W. N. Li, "On some new nonlinear discrete inequalities and their applications," Journal of Computational and Applied Mathematics, vol. 158, no. 2, pp. 407-417, 2003.

[21] W. S. Cheung, "Some discrete nonlinear inequalities and applications to boundary value problems for difference equations," Journal of Difference Equations and Applications, vol. 10, no. 2, pp. 213-223, 2004.

[22] F. W. Meng and D. H. Ji, “On some new nonlinear discrete inequalities and their applications," Journal of Computational and Applied Mathematics, vol. 208, no. 2, pp. 425-433, 2007.

[23] W. S. Cheung and Q. H. Ma, "On certain new Gronwall-Ou-Iang type integral inequalities in two variables and their applications," Journal of Inequalities and Applications, vol. 2005, no. 4, pp. 347-361, 2005.

[24] W. S. Cheung and J. L. Ren, "Discrete non-linear inequalities and applications to boundary value problems," Journal of Mathematical Analysis and Applications, vol. 319, no. 2, pp. 708-724, 2006.

[25] A. Gallo and A. M. Piccirillo, "About some new generalizations of Bellman-Bihari results for integrofunctional inequalities with discontinuous functions and applications," Nonlinear Analysis: Theory, Methods \& Applications, vol. 71, no. 12, pp. e2276-e2287, 2009.

[26] R. A. C. Ferreira and D. F. M. Torres, "Generalized retarded integral inequalities," Applied Mathematics Letters, vol. 22, no. 6, pp. 876-881, 2009.

[27] F. W. Meng and W. N. Li, "On some new integral inequalities and their applications," Applied Mathematics and Computation, vol. 148, no. 2, pp. 381-392, 2004.

[28] R. Xu and Y. G. Sun, "On retarded integral inequalities in two independent variables and their applications," Applied Mathematics and Computation, vol. 182, no. 2, pp. 1260-1266, 2006.

[29] Z. L. Yuan, X. W. Yuan, and F. W. Meng, "Some new delay integral inequalities and their applications," Applied Mathematics and Computation, vol. 208, no. 1, pp. 231-237, 2009.

[30] R. P. Agarwal, S. F. Deng, and W. N. Zhang, "Generalization of a retarded Gronwall-like inequality and its applications," Applied Mathematics and Computation, vol. 165, no. 3, pp. 599-612, 2005. 


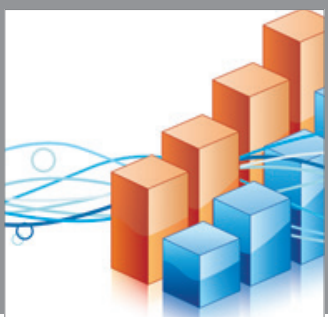

Advances in

Operations Research

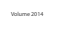

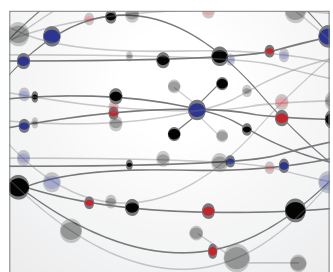

\section{The Scientific} World Journal
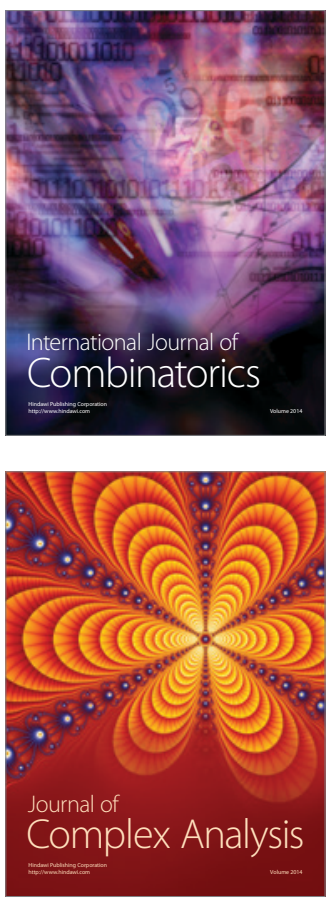

International Journal of

Mathematics and

Mathematical

Sciences
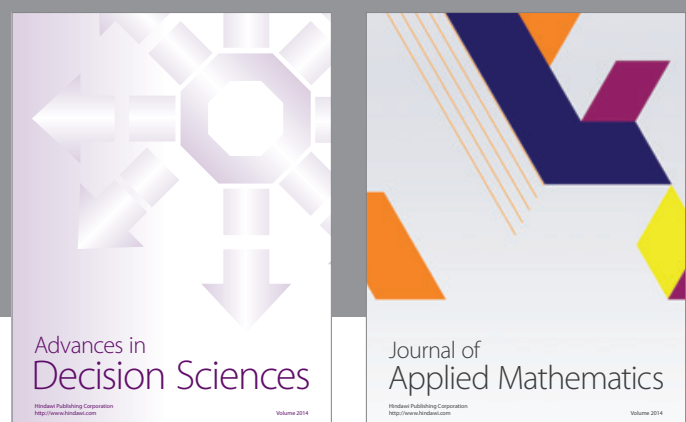

Journal of

Applied Mathematics
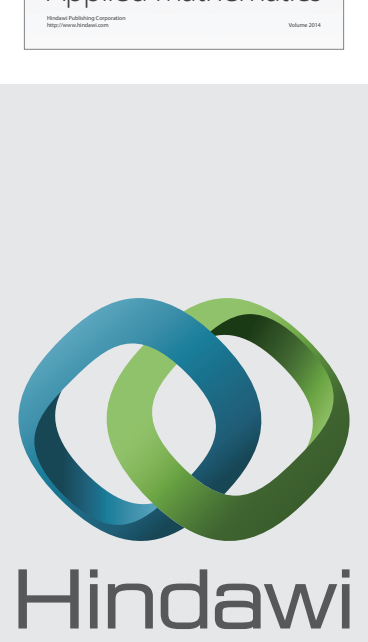

Submit your manuscripts at http://www.hindawi.com
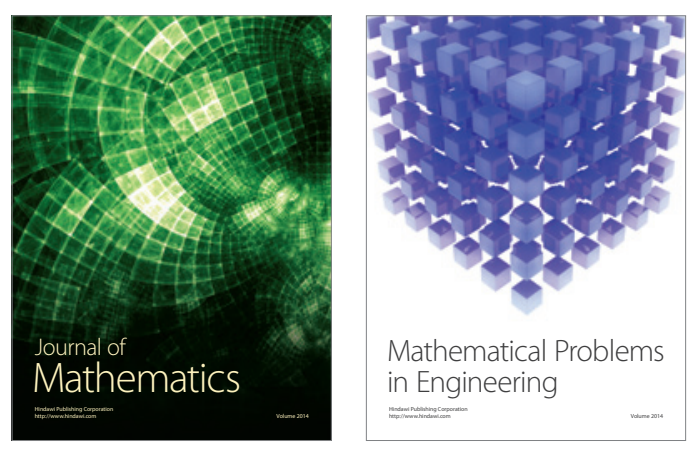

Mathematical Problems in Engineering
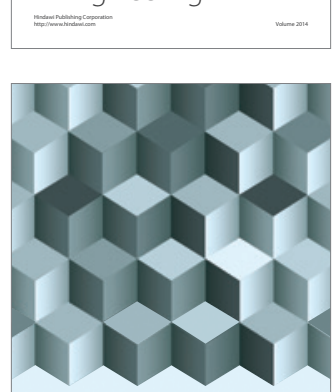

Journal of

Function Spaces
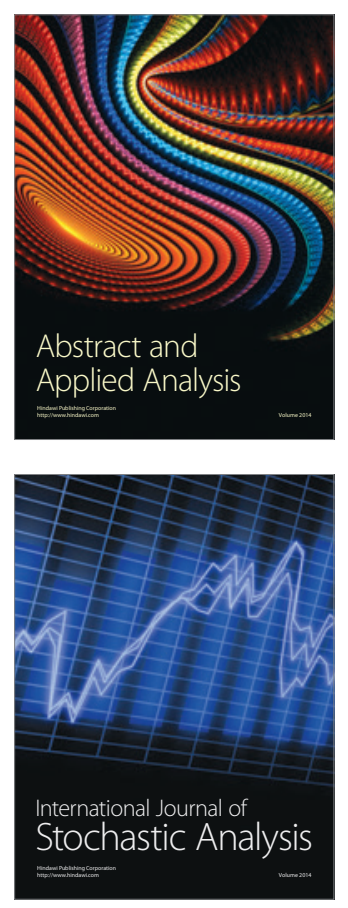

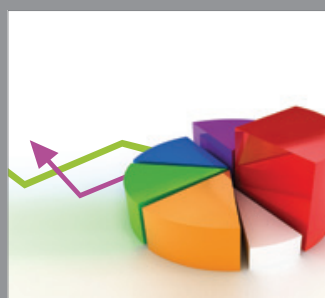

ournal of

Probability and Statistics

Promensencen
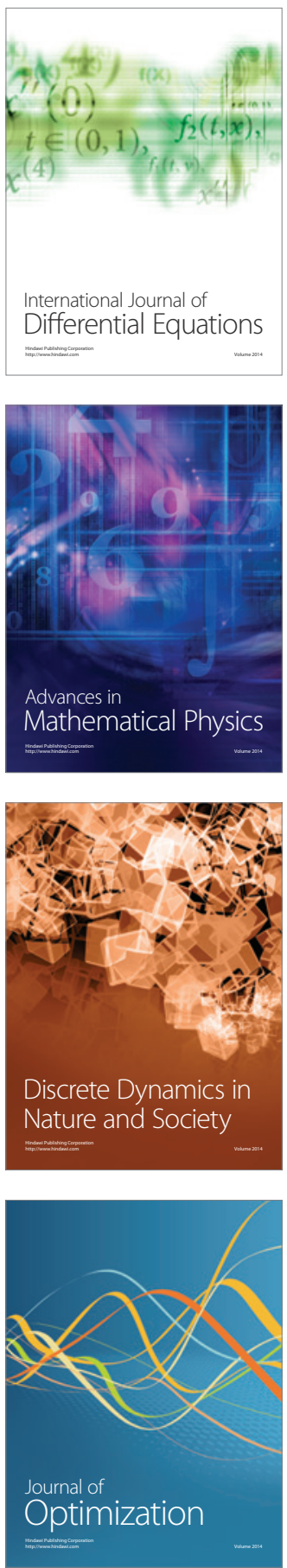